\title{
Un modelo alométrico
}

\section{pantropical global y local}

\section{A global and local allometric pantropical model}

\author{
Fernando Paz-Pellat ${ }^{1 *}$ \\ GRENASER, Colegio de Postgraduados. Campus \\ * Autor de correspondencia: ferpazpel@gmail.com \\ Montecillo. Montecillo, Estado de México, México.
}

\section{RESUMEN}

El desarrollo de modelos alométricos generalizados que permitan realizar estimaciones comparables con modelos locales, es un gran reto para la realización de estimaciones de la biomasa aérea en los bosques tropicales. Las estimaciones de los modelos alométricos parametrizados en el espacio logarítmico (transformación a formato logarítmico) minimizando el error cuadrático de estimación requieren de la estimación de factores de corrección para la transformación inversa al espacio aritmético. Adicionalmente, si el objetivo es la minimización de sesgos (error relativo medio, ERM y error absoluto medio, EAM), entonces se puede minimizar el error absoluto de estimación. En este trabajo se usaron modelos alométricos clásicos, basados en la relación entre la biomasa (B) y el diámetro normal (D), altura total (H) y densidad de la madera ( $\rho)$, para revisar las relaciones entre sus parámetros. Para analizar las relaciones alométricas planteadas se utilizó una base de datos pública pantropical (4004 datos, 58 sitios de muestreo). Los análisis mostraron que para modelos globales (todos los sitios) y locales (cada sitio) el modelo de regresión lineal de la relación B versus $\mathrm{\rho D}_{2} \mathrm{H}$ resultó en el mejor modelo (métrica de la raíz del error cuadrático medio o RECM), por ello fue usado como estándar de referencia. Los modelos parametrizados en el espacio logarítmico para las estimaciones globales resultaron con errores de estimación mayores al modelo $\mathrm{B}=a_{\mathrm{v} 0}\left(\mathrm{D}^{2} \mathrm{H}\right)$ con $a_{\mathrm{v} 0}$ como función lineal con $\rho$. La estimación de $a_{\mathrm{v} 0}$ fue realizada minimizando el error absoluto, resultando en los menores errores de sesgos de estimación (EAR y EAM), con valores del RECM comparables al proceso de minimización del error cuadrático. Para las estimaciones locales, usando modelos alométricos a nivel de sitio, se utilizó el modelo con solo $a_{\mathrm{v} 0}$ (minimización del error absoluto) y cambiando el factor de corrección del estimador simple al de razones, resultando en un modelo de predicción con error de estimación comparables al de las regresiones no lineales y superando los modelos de alometría clásicos. Dado que no se cuenta con información de la biomasa aérea en los inventarios forestales normales, la estimación del factor de corrección de razones fue parametrizado en forma empírica por un proceso de regresión lineal multivariada de datos medidos en campo con resultados comparables a contar con mediciones de campo de la biomasa aérea.

PAlABRAS ClAVE: alometría condicionada a campo, densidad de madera, errores de estimación, factores de corrección, minimización del error absoluto.

\section{ABSTRACT}

The development of generalized allometric models that allow estimations that are comparable with local models is a great challenge for estimating aerial biomass in tropical forests. The estimates of the parametrized allometric models in the logarithmic space (transformation to logarithmic format) minimizing the squared error of estimation requires the estimation of correction factors for the inverse transformation to the arithmetic space. Additionally, if the objective is the minimization of biases (mean relative error MRE and mean absolute error MAE), then the absolute estimation error can be minimized. In this work, classic allometric models were used, based on the relationship between biomass (B) and normal diameter $(\mathrm{D})$, total height $(\mathrm{H})$ and wood density $(\rho)$, to review the relationships between their parameters. To analyze the proposed allometric relationships, a pantropical public database (4 004 data, 58 sampling sites) was used. The analyzes showed that for global models (all sites) and local (each site) the linear regression model of the relationship B versus $\rho \mathrm{D}^{2} \mathrm{H}$ resulted in the best model (root mean square error or RMSE metric), for which was used as a reference standard. The models parametrized in the logarithmic space for the global estimates resulted with estimation errors greater than the model $\mathrm{B}=a_{\mathrm{v} 0}\left(\mathrm{D}^{2} \mathrm{H}\right)$ with $a_{\mathrm{v} 0}$ as a linear function with $\rho$. The estimation of $a_{\mathrm{v} 0}$ was performed by minimizing the absolute error, resulting in the lowest estimation bias errors (MRE and MAE), with RMSE values comparable to the quadratic error minimization process. For local estimates using allometric models at the site level, the model was used with only $a_{\mathrm{v} 0}$ (minimization of the absolute error) and changing the correction factor from the simple estimator to that of ratio estimator, resulting in a prediction model with an estimation error comparable to the nonlinear regressions and surpassing the classic allometry models. Since there is no information on aerial biomass in normal forest inventories, the estimation of the ratio correction factor was empirically parameterized by a multivariate linear regression process of data measured in the field, with results comparable to having measurements of aerial biomass on the field.

KEYWORDS: absolute error minimization, correction factors, estimation errors, field-conditioned allometry, wood density. 


\section{INTRODUCCIÓN}

Uno de los grandes retos del sector forestal con relación a su manejo para mitigar los impactos del cambio climático es la necesidad de conocer los almacenes de carbono de estos ecosistemas. En lo particular, la biomasa aérea de los bosques requiere del uso de ecuaciones alométricas (Brown, 1997), donde las variables diámetro a la altura del pecho $(1.3 \mathrm{~m})$ o diámetro normal $(\mathrm{D})$, altura total de los árboles $(\mathrm{H})$ y densidad de la madera $(\rho)$ son utilizadas normalmente en forma individual o combinada (Brown, 1997; Chave et al., 2005; Vargas-Larreta et al., 2017). La selección del modelo alométrico es una de las principales fuentes de incertidumbre en la propagación de los errores de estimación de la biomasa aérea (B) (Pelletier, Kirby y Potvin, 2012; Picard, Boyemba y Rossi, 2015).

Para las estimaciones confiables de la biomasa aérea de especies de árboles en un determinado sitio es necesario el conocimiento de la ecuación alométrica de cada especie en el área de análisis. Esto conlleva a contar con un catálogo de ecuaciones (Jenkins, Chojncky, Heath y Birdsey, 2003; Henry et al., 2011; Rojas-García, de Jong, Martínez-Zurimendi y Paz-Pellat, 2015) para la selección adecuada. Evidentemente este esquema es costoso en tiempo y en recursos, además de que no garantiza que la ecuación seleccionada para una especie sea representativa de la población particular en análisis (p. ej. Méndez González, Turlan Medina, Ríos Saucedo y Nájera Luna, 2012), ya que los parámetros de los modelos alométricos son función del tipo de vegetación, clima, estructura de la población, arquitectura de las plantas, condición del sitio de muestreo, ontogenia, entre otros factores (Wutzler, Wirth y Schumacher, 2008; Genet et al., 2011; Chave et al., 2014; Paul et al., 2016; Forrester et al., 2017). El uso de un modelo alométrico en un sitio diferente al que fue desarrollado conlleva alta incertidumbre no cuantificada en las estimaciones (Jenkins et al., 2003; Temesgen, Affleck, Poudel, Gray y Sessions, 2015), por lo que se debe tener cuidado en la implementación a ciegas de este enfoque.

Una alternativa a la complejidad del problema que ha sido explorada es el desarrollo de modelos generalizados por tipo de ecosistema o de tipo pantropical (p. ej. Chave et al., 2005 y 2014) que consiste en la recopilación de datos medidos en campo y laboratorio de sitios alrededor del mundo, donde cada sitio consta de un conjunto de especies característico del ecosistema terrestre en evaluación. El modelo generalizado obtenido se espera que sea representativo del ecosistema y que genere estimaciones no sesgadas y precisas. Este enfoque ha sido seriamente cuestionado con relación al uso de modelos locales (a nivel de sitio), que generalmente realizan mejores estimaciones (Basuki, Vaan Laake, Skidmore y Hussin; 2009; Henry et al.; 2011; Van Breugel, Ransijn, Craven, Bongers y Hall, 2011; Álvarez et al.; 2012; Ngomanda et al.; 2014; Sato et al.; 2015; Manuri et al., 2016; Ploton et al., 2016). Lo ideal es el desarrollo de un modelo alométrico adaptativo que considere datos locales $(\mathrm{D}, \mathrm{H}, \rho)$ para las estimaciones de la biomasa aérea (B) y que sea comparable al uso de ecuaciones alométricas locales con relación a la incertidumbre de estas.

Un problema asociado al desarrollo de modelos alométricos tipo $\mathrm{Y}=a \mathrm{X}^{b}$, es el proceso de estimación de sus parámetros. La práctica común es transformar el espacio aritmético a uno logarítmico, $\operatorname{Ln}(\mathrm{Y})=\operatorname{Ln}(a)+b \operatorname{Ln}(\mathrm{X})$, para estimar $a$ y $b$ usando regresión (ordinaria) lineal simple, minimizando el error cuadrático de estimación. La transformación inversa del espacio logarítmico al aritmético introduce sesgos que requieren ser corregidos (Zar, 1968). Al respecto, existen diferentes estimadores para corregir los sesgos fundamentados en diferentes hipótesis y modelos (Finney, 1941; Bradu y Mundlak, 1970; Baskerville, 1972; Beauchamp y Olson, 1973; Duan, 1983; Snowdon, 1991; El-Shaarawi y Viveros, 1997; Shen y Zhu, 2008). En el proceso de evaluación de los métodos de corrección de sesgos (Lee, 1982; Smith, 1993; Hui, Terblance, Chown y McGeoch, 2010; Zeng y Tang, 2011; Clifford, Cressie, England, Roxburgh y Paul, 2013) se han encontrado resultados mixtos dependientes del grado en que los datos representan las hipótesis utilizadas. En esta perspectiva, se ha argumentado que la mejor opción para evitar la incertidumbre de los métodos de corrección de 
sesgos es el uso de técnicas de regresión no lineal aplicada directamente en el espacio aritmético (Packard, 2017).

\section{OBJETIVOS}

En este trabajo se analizan los enfoques típicos de desarrollo de modelos alométricos clásicos, así como la corrección de sesgos más utilizada, con el objetivo de desarrollar un modelo a escala global (multiespecie y multisitio) que puede ser parametrizado empíricamente a escala local (sitio y multiespecie), aproximando el ideal del uso de alometría en bosques tropicales.

\section{MATERIALES Y MÉTODOS}

\section{Modelos alométricos utilizados}

Para posicionar el problema de estimación usando modelos alométricos de la biomasa aérea, esta puede ser puesta como $\mathrm{B}=\rho \mathrm{V}$ (Cannell, 1984), donde $\mathrm{V}$ es el volumen que puede ser aproximado por el volumen de un fuste cilíndrico, $V=(\pi / 4)\left(D^{2} H\right)$, con un factor de corrección para otras geometrías, además de la consideración del volumen de las ramas y hojas de la corona del árbol usando un factor de expansión de la biomasa. Este enfoque geométrico plantea una ecuación del tipo $\mathrm{B}=a_{\mathrm{c}}\left(\rho \mathrm{D}^{2} \mathrm{H}\right)$, donde $a_{\mathrm{c}}$ es un factor de corrección general (forma del fuste y volumen de la corona y sus componentes). La densidad de la madera se considera como representativa de todas las componentes estructurales de los árboles. En la perspectiva discutida, los modelos alométricos considerados son:

$$
\begin{aligned}
& M 1: B=a_{v}\left(D^{2} H\right)^{b_{v}} \\
& M 2: B=a_{v d}\left(\rho D^{2} H\right)^{b_{v d}}=a_{v d} \rho^{b_{v d}}\left(D^{2} H\right)^{b_{v d}} \\
& M 3: B=a_{v 0}\left(D^{2} H\right)
\end{aligned}
$$

donde $a$ y $b$ representan constantes del ajuste estadístico.

Usando el modelo M2 como referencia, comparándolo con el modelo M1 y M3, se obtiene:

$$
\begin{aligned}
& a_{v}=a_{v d} \rho^{b_{v d}} y b_{v}=b_{v d} \\
& a_{v 0}=a_{v d} \rho \text { y } b_{v d}=1
\end{aligned}
$$

En términos algebraicos, el modelo M1 y M2 son equivalentes entre sí por la relación (4) de sus parámetros. En el caso del modelo M3, la equivalencia entre parámetros está dada por la relación (5), para el caso de $b_{\mathrm{vd}}=b_{\mathrm{v}}=1$.

\section{Estimación de los parámetros de los modelos alométricos}

La ecuación alométrica dada por:

$$
Y=a X^{b} \varepsilon
$$

puede transformarse logarítmicamente como:

$$
\operatorname{Ln}(Y)=Y^{\prime}=a+b \operatorname{Ln}(X)+\operatorname{Ln}(\varepsilon)
$$

para estimar los parámetros $a$ y $b$ por regresión lineal simple, donde $\varepsilon$ es el error de estimación [Y'medido Y'estimado].

Aunque hay diferentes estimadores del factor de corrección de la transformación inversa del espacio logarítmico al aritmético, el estimador simple o ES ("naive estimator", Duan, 1983) de Baskerville (1972) es el más utilizado:

$$
\begin{aligned}
& Y=\operatorname{Exp}\left[\frac{\sigma}{2}+a+b \operatorname{Ln}(X)\right] \\
& Y=\left(a X^{b}\right) \mathrm{FC}_{\mathrm{ES}}
\end{aligned}
$$

donde $\mathrm{FC}_{\mathrm{ES}}=\operatorname{Exp}(\sigma / 2)$ es el factor de corrección del estimador simple y $\sigma$ es la desviación estándar (error estándar residual o EER) del error $\varepsilon$, el cual es supuesto como distribuido normalmente con media cero y desviación estándar $\sigma$, definida como:

$$
\sigma=E E R=\sqrt{\frac{1}{n-p} \sum_{i=1}^{n}\left(Y_{i}^{\prime}-\hat{Y}_{i}^{\prime}\right)^{2}}
$$


donde $\hat{Y}_{i}^{\prime}$ es el valor estimado, $Y_{i}^{\prime}$ el valor medido, $\mathrm{n}$ el número de datos y $\mathrm{p}$ los parámetros del modelo $(\mathrm{p}=2$ para los modelos M1 y M2).

Otro estimador utilizado (e.g. Búrquez y Martínez-Yrízar, 2011) es el estimador de razón (ER) de Snowdon (1991):

$$
Y=\left(a X^{b}\right) \mathrm{FC}_{\mathrm{ER}}
$$

donde $\mathrm{FC}_{\mathrm{ER}}=$ Promedio $($ Bmed $) /$ Promedio $($ Best $)$, donde Best es obtenida de la aplicación del modelo alométrico en el espacio logarítmico, sin realizar ninguna corrección, y el término est se refiere a estimada y med a medida.

El ajuste de los modelos alométricos se analizó a través del uso de diferentes métricas de incertidumbre, incluyendo el coeficiente de determinación $\left(\mathrm{R}^{2}\right)$, como la raíz del error cuadrático medio (RECM):

$$
R E C M=\sqrt{\frac{1}{n} \sum_{i=1}^{n}\left(Y_{i}^{\prime}-\hat{Y}_{i}^{\prime}\right)^{2}}
$$

y el error relativo medio (ERM) y error absoluto medio (EAM), ambos en porcentaje:

$$
\begin{aligned}
& E R M=100\left\{\frac{1}{n} \sum_{i=1}^{n} \frac{Y_{i}^{\prime}-\hat{Y}_{i}^{\prime}}{Y_{i}^{\prime}}\right\} \\
& E A M=100\left\{\frac{1}{n} \sum_{i=1}^{n}\left|\frac{Y_{i}^{\prime}-\hat{Y}_{i}^{\prime}}{Y_{i}^{\prime}}\right|\right\}
\end{aligned}
$$

Los resultados del proceso de estimación pueden ser analizados con relación entre lo medido (med) y lo estimado (est):

$$
\begin{aligned}
& \operatorname{Ln}(Y) e s t=q+r \operatorname{Ln}(Y) \text { med } \\
& \text { Yest }=s+t Y \text { med }
\end{aligned}
$$

donde para una estimación perfecta se espera que $q$ y $s$ sean igual a 0.0 y los parámetros $r$ y $s$ igual a 1.0.
La estimación de los parámetros de los modelos alométricos generalmente se obtiene por un proceso de regresión lineal simple al minimizar el error cuadrático de estimación $\left(\varepsilon^{2}\right)$, lo cual implica una simetría (término cuadrático) que no necesariamente es la mejor opción de estimación. Una alternativa de estimación es minimizar el error absoluto de estimación (|c|) (Journel, 1984).

\section{Base de datos de alometría pantropical analizada}

Para tener un base de datos representativa de los bosques tropicales en el mundo, se analizó la publicada por Chave et al. (2014), disponible públicamente, la cual consiste en 4004 mediciones de $\mathrm{D}(\mathrm{cm}), \mathrm{H}(\mathrm{m})$ y $\rho\left(\mathrm{g} \mathrm{cm}^{-3}\right)$ en 58 sitios en diferentes partes del mundo, donde 53 sitios son de vegetación no perturbada. La densidad de la madera de la base de datos fue medida o estimada; en un $58 \%$ de los datos se midió y en el resto fue estimada por el valor promedio de la especie, género o familia usando una base de datos global (Chave et al., 2009; Zanne et al., 2009). La documentación de los sitios y de la base de datos se encuentra en Chave et al. (2014), por lo que solo se presenta una descripción mínima en este trabajo. Adicionalmente, Burt et al. (2020) analizaron la base de datos con relación a errores, y discuten sus implicaciones y limitaciones.

Las unidades de las variables de la base de datos son las mismas que las usadas en todos los análisis presentados en este trabajo, por lo que no serán incluidas en lo siguiente.

En la tabla 1 se muestran los sitios incluidos en la base de datos, además del número de datos y el valor máximo de $\mathrm{D}$ en cada sitio.

La figura 1 muestra la relación entre la biomasa $\mathrm{B}$ con relación a $\rho \mathrm{D}^{2} \mathrm{H}$, donde se muestra una gran dispersión, por lo que el uso de un modelo alométrico generalizado tendrá limitaciones en explicar la variabilidad observada.

\section{Posicionamiento del problema}

Para posicionar el problema planteado en este trabajo, la figura 2 muestra los modelos alométricos (M2) locales 
(58) ajustados por regresión no lineal a la base de datos, además del modelo global (curva punteada). En la figura se observa que el modelo global solo aproxima algunos modelos locales, por lo que en muchos casos se sobre o subestima con relación a los modelos alométricos de sitios particulares. La solución al modelo planteado de una solución general que sea aplicable a nivel local requiere redefinir el problema de estimación.

TABLA 1. Sitios y características generales incluidos en la base de datos.

\begin{tabular}{|c|c|c|c|c|c|c|c|c|}
\hline Sitio & $n$ & D máx. (cm) & Sitio & $n$ & D máx. $(\mathrm{cm})$ & Sitio & $n$ & D máx. (cm) \\
\hline Australia & 46 & 24.9 & Kalimanı & 23 & 77.6 & PuertoRi & 30 & 45.7 \\
\hline BraMan2 & 123 & 38.2 & Kaliman2 & 69 & 130.5 & PuertoRi2 & 25 & 45.0 \\
\hline BraParal & 127 & 138.0 & Kaliman4 & 40 & 68.9 & SaoPaulo3 & 75 & 67.8 \\
\hline BraPara3 & 21 & 55.0 & Kaliman6 & 25 & 84.4 & Sarawak & 21 & 44.1 \\
\hline BraRond & 8 & 89.0 & Karnataka & 189 & 60.9 & SouthAfrica & 469 & 79.3 \\
\hline Cambodia & 34 & 133.2 & Llanosec & 24 & 23.3 & SouthBrazill & 150 & 95.0 \\
\hline Cameroon & 5 & 79.4 & Llanosol & 27 & 156.0 & SouthBrazill & 50 & 124.8 \\
\hline Cameroon3 & 59 & 212.0 & Madagascarl & 76 & 54.0 & SouthBrazil3 & 64 & 34.5 \\
\hline CentralAfric & 12 & 52.2 & Madagascar2 & 90 & 35.0 & Sumatra & 29 & 48.1 \\
\hline ColombiaCl & 60 & 126.7 & Madagascar3 & 87 & 31.8 & Sumatra2 & 11 & 114.6 \\
\hline ColombiaGl & 36 & 70.9 & Madagascar4 & 80 & 37.0 & Tanzanial & 38 & 78.0 \\
\hline ColombiaG2 & 10 & 12.5 & Madagascar5 & 90 & 36.0 & Tanzania2 & 42 & 110.0 \\
\hline ColombiaM1 & 24 & 111.9 & Malaysia & 139 & 101.6 & Tanzania3 & 38 & 79.0 \\
\hline ColombiaM2 & 9 & 11.8 & Malaysia2 & 24 & 66.7 & Tanzania4 & 34 & 95.0 \\
\hline CostaRic & 97 & 116.0 & MFrenchG & 29 & 42.0 & Venezuela2 & 40 & 136.8 \\
\hline FrenchGu & 360 & 117.8 & MGuadel & 55 & 40.7 & WestJava & 41 & 31.8 \\
\hline Gabon & 103 & 109.4 & Moluccas & 25 & 41.7 & Yucatan & 175 & 63.4 \\
\hline Ghana & 37 & 180.0 & Mozambique & 28 & 72.0 & Zambia & 141 & 37.4 \\
\hline IndiaCha & 23 & 34.7 & NewGuinea & 42 & 110.1 & & & \\
\hline Jalisco & 124 & 44.9 & Peru & 51 & 169.0 & & & \\
\hline
\end{tabular}




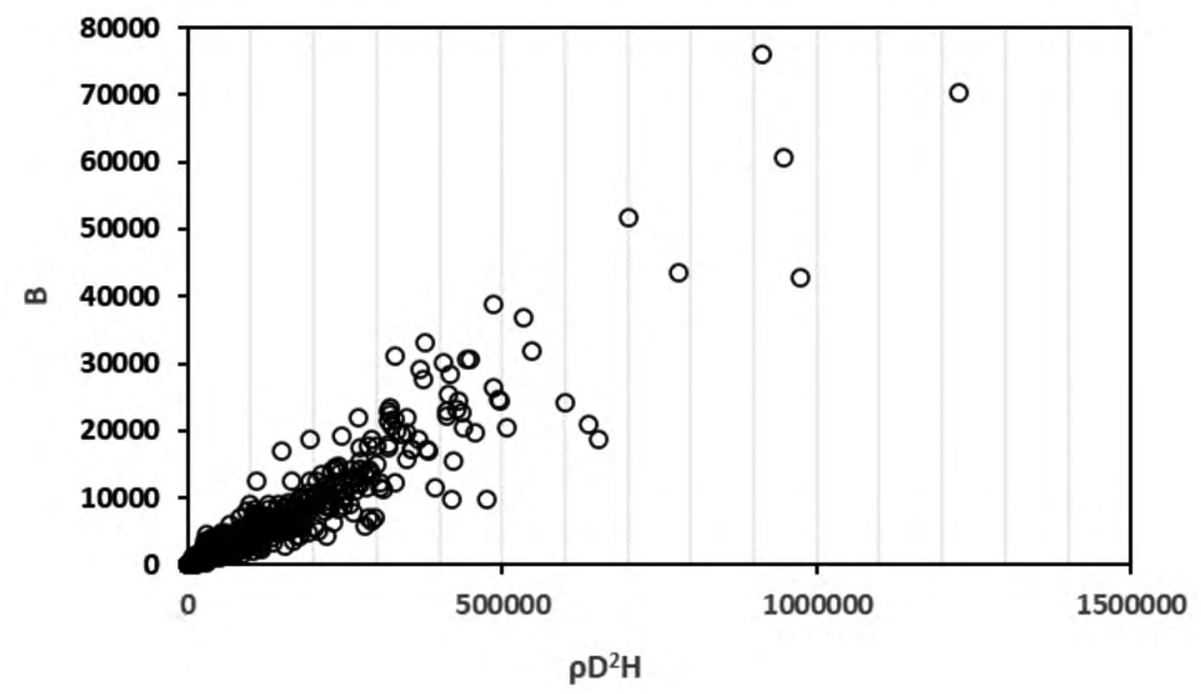

Figura 1. Relación entre la biomasa $\mathrm{B}$ y $\rho \mathrm{D}^{2} \mathrm{H}$ para todos los sitios de la base de datos pantropical.
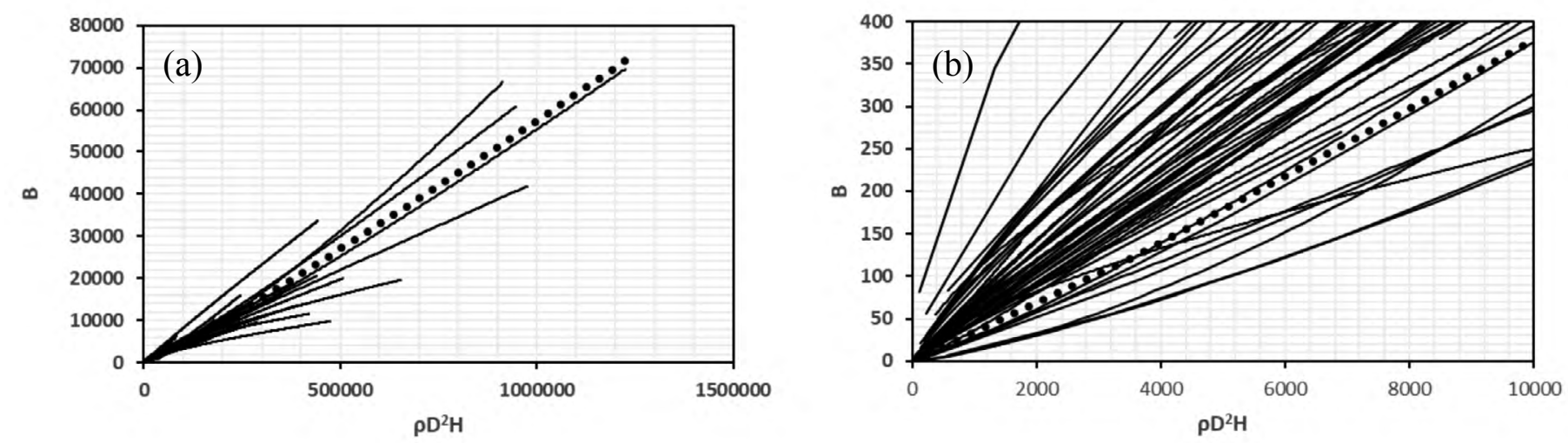

Figura 2. Modelos alométricos ajustados por regresión no lineal a cada sitio de la base de datos, además del modelo global (curva punteada). (a) Valores generales y, (b) valores pequeños de $\rho \mathrm{D}^{2} \mathrm{H}$.

\section{RESULTADOS}

Los ajustes por regresión estadística fueron realizados usando la función Solver ${ }^{\mathrm{MR}}$ de Excel $^{\mathrm{MR}}$, minimizando el error cuadrático de estimación.

\section{Modelos alométricos globales}

Los resultados de los ajustes estadísticos están mostrados en la tabla 2 para el espacio logarítmico y la tabla 3 para el aritmético. En el modelo M3 de la relación (3), este representa el caso donde $a_{\mathrm{v} 0}$ fue estimada por regresión lineal en el espacio logarítmico y el modelo $3 \mathrm{~b}$ utiliza la relación (5) para el modelo $\mathrm{M} 3$, donde $\rho$ representa valores específicos en cada sitio de la base datos y el valor $a_{\mathrm{vd}}$ $=0.0524$ fue estimado en el análisis realizado. El modelo M2a (Chave et al., 2014) fue estimado usando regresión lineal en el espacio logarítmico y el modelo M2b fue parametrizado por regresión no lineal directamente en el espacio aritmético y los parámetros mostrados son simples conversiones al espacio logarítmico $(\mathrm{FC}=1.0)$. Para el espacio aritmético, la conversión utilizó $\mathrm{FC}_{\mathrm{ES}}$. 
TABLA 2. Parámetros y estadísticos de los ajustes en el espacio logarítmico de modelos globales.

\begin{tabular}{llllllll}
\hline Modelo & Parámetros & $q$ & $r$ & $R^{2}$ & ERM & EAM & RECM \\
\hline M1 & $\operatorname{Ln}\left(a_{\mathrm{v}}\right)=-3.0626, b_{\mathrm{v}}=0.9535$ & 0.1954 & 0.9590 & 0.9590 & -2.4131 & 10.1037 & 0.4294 \\
M3a & $\operatorname{Ln}\left(a_{\mathrm{v}}\right)=-3.4443, b_{\mathrm{v}}=1$ & -0.0274 & 1.0057 & 0.9590 & -1.0066 & 10.0904 & 0.4412 \\
M3b & $\operatorname{Ln}\left(a_{\mathrm{vo}}\right)=\operatorname{Ln}(0.0524 \rho), b_{\mathrm{v}}=1$ & 0.0210 & 0.9956 & 0.9716 & -0.7326 & 7.9350 & 0.3612 \\
M2a & $\operatorname{Ln}\left(a_{\mathrm{vd}}\right)=-2.7628, b_{\mathrm{vd}}=0.9759$ & -0.1354 & 0.9716 & 0.9716 & -1.4706 & 8.0312 & 0.3575 \\
M2b & $\operatorname{Ln}\left(a_{\mathrm{vd}}\right)=\operatorname{Ln}(0.0164), b_{\mathrm{vd}}=1.0906$ & -0.8703 & 1.0858 & 0.9716 & 14.7458 & 15.9937 & 0.6333 \\
\hline
\end{tabular}

TABLA 3. Parámetros y estadísticos de los ajustes en el espacio aritmético de modelos globales.

\begin{tabular}{lllllllll}
\hline Modelo & Parámetros & $F C_{E S}$ & $s$ & $t$ & $R^{2}$ & $E R M$ & $E A M$ & $R E C M$ \\
\hline M1 & $\mathrm{a}_{\mathrm{v}}=\operatorname{Exp}(-3.0626), \mathrm{b}_{\mathrm{v}}=0.9535$ & 1.0966 & 206.88 & 0.8278 & 0.8259 & -21.2015 & 41.0710 & 1634.8729 \\
M3a & $\mathrm{a}_{\mathrm{v}}=\operatorname{Exp}(-3.4443), \mathrm{b}_{\mathrm{v}}=1$ & 1.1023 & 185.30 & 1.0675 & 0.8246 & -22.3489 & 42.4035 & 1964.1605 \\
M3b & $\mathrm{a}_{\mathrm{vo}}=0.0524 \rho, \mathrm{b}_{\mathrm{v}}=1$ & 1.0674 & 153.50 & 0.9641 & 0.9116 & -13.9983 & 31.5284 & 1190.0537 \\
M2a & $\mathrm{a}_{\mathrm{vd}}=\operatorname{Exp}(-2.7628), \mathrm{b}_{\mathrm{vd}}=0.9759$ & 1.0660 & 168.60 & 0.8478 & 0.9091 & -13.7583 & 31.3052 & 1207.8154 \\
M2b & $\mathrm{a}_{\mathrm{vd}}=0.0164, \mathrm{~b}_{\mathrm{vd}}=1.0906$ & & 27.82 & 0.9205 & 0.9161 & 30.7668 & 38.5995 & 1136.3307 \\
\hline
\end{tabular}

En la figura 3 se muestran los resultados espacio logarítmico y aritmético de los modelos M2a (Chave et al., 2014) y el modelo M2b de la regresión no lineal. De la tabla 3, el modelo de regresión no lineal es el mejor modelo usando el criterio de la métrica RECM, la cual es la única relevante, dado que el proceso de regresión, lineal y no lineal busca minimizarla. Las otras métricas de error son estimadas como consecuencia del proceso de minimización del error cuadrático. De las tablas 2 y 3 , tener un error menor en el espacio logarítmico no implica que al transformar el modelo al espacio aritmético este siga teniendo un error menor. El caso del modelo M2b (regresión no lineal) ejemplifica esta situación.

El modelo M1 (no inclusión de $\rho$ ) muestra resultados pobres con relación a los otros modelos analizados, mostrando que el no considerar la densidad de la madera produce resultados con errores mayores a los de su consideración, algo similar ocurre con el modelo M3a. Ahora bien, el caso del modelo M3b (inclusión de $\rho$ a nivel de sitio) (Fig. 4) que utiliza la hipótesis de que $b_{\text {vd }}=1$, mues- tra estadísticos de errores menores que el caso del modelo de Chave et al. (2014) (Tabla 2 y 3), que además reduce la dimensionalidad del problema (modelo con un solo parámetro).

\section{Modelos alométricos locales}

Para el caso de los ajustes de modelos alométricos locales (en cada sitio), la tabla 4 (espacio logarítmico) y tabla 5 (espacio aritmético) muestran los resultados obtenidos, donde el mejor ajuste fue el modelo M2b (regresión no lineal) y después el modelo M2a de regresión lineal en el espacio logarítmico (Fig. 5). El modelo M3b, que considera la densidad de la madera a nivel local (sitio), mostró un comportamiento ligeramente no mejor que el caso global.

En la figura 5 y de la tabla 4 se observa que el mejor modelo (M2b) muestra los peores resultados en el espacio logarítmico, por lo que es necesario considerar la inversión de los modelos del espacio logarítmico al aritmético, ya que estos están orientados a la minimización de errores en el formato logarítmico que, si no se usa un factor de correc- 
ción adecuado, pueden tener errores grandes de estimación en el espacio aritmético.

\section{Minimización del error absoluto}

Para el caso del modelo M3b se realizó un proceso de minimización del error absoluto (EAM), obteniéndose resulta- dos en el espacio logarítmico con el menor sesgo (ERM y EAM), con resultados mejores de RECM a los de la minimización del error cuadrático. La figura 6 muestra los resultados obtenidos. El modelo fue parametrizado con el valor $a_{\mathrm{v} 0}=0.0595$, cuyo valor será utilizado en lo siguiente.
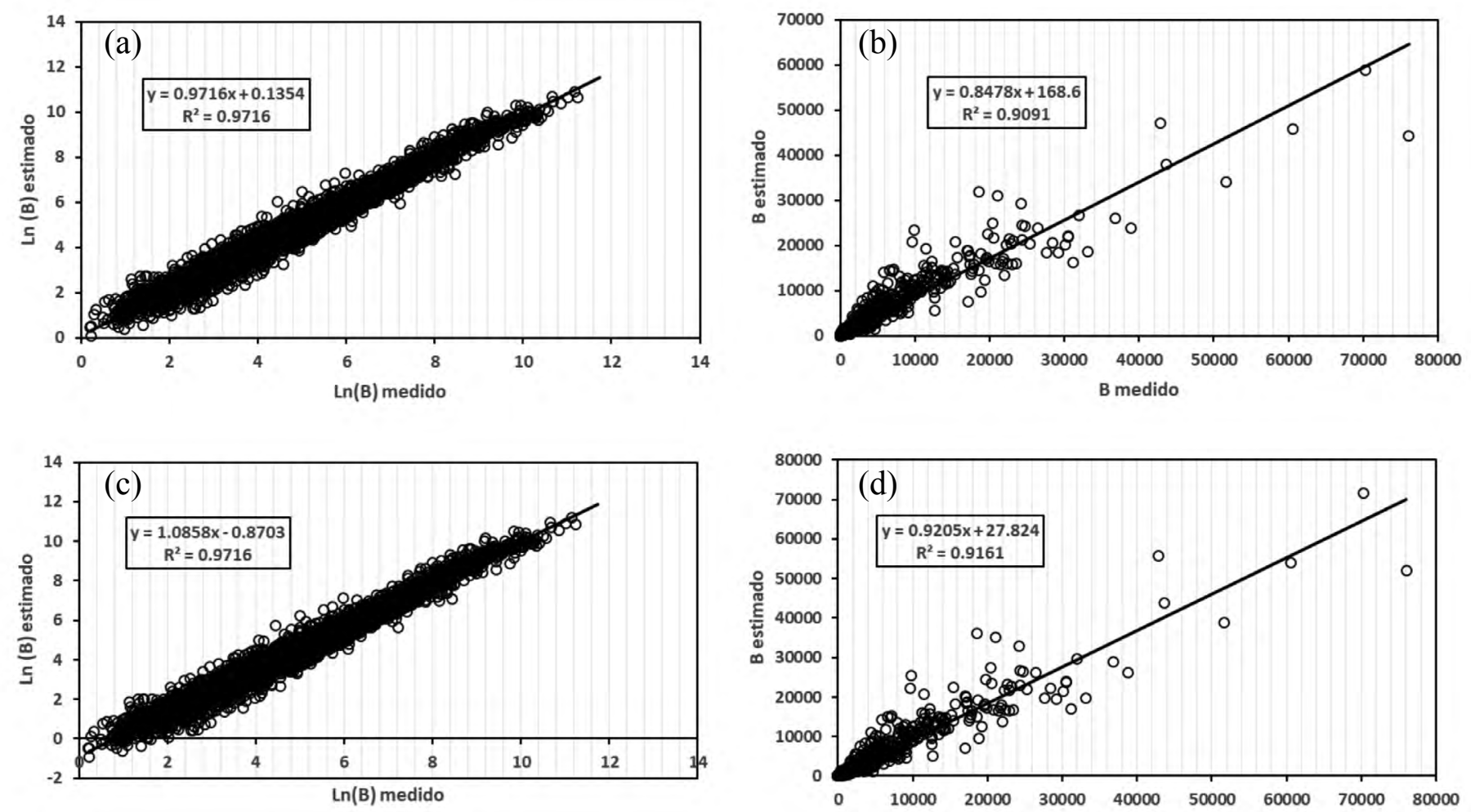

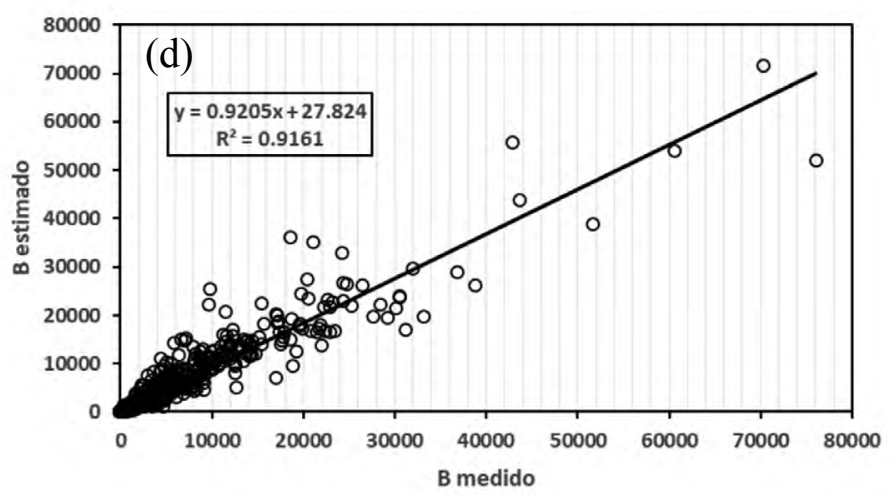

Figura 3. Resultados del proceso de estimación de modelos globales. (a) Modelo M2a en espacio logarítmico, (b) Modelo M2a en espacio aritmético, (c) Modelo M2b en espacio logarítmico y (d) Modelo M2b en espacio aritmético.
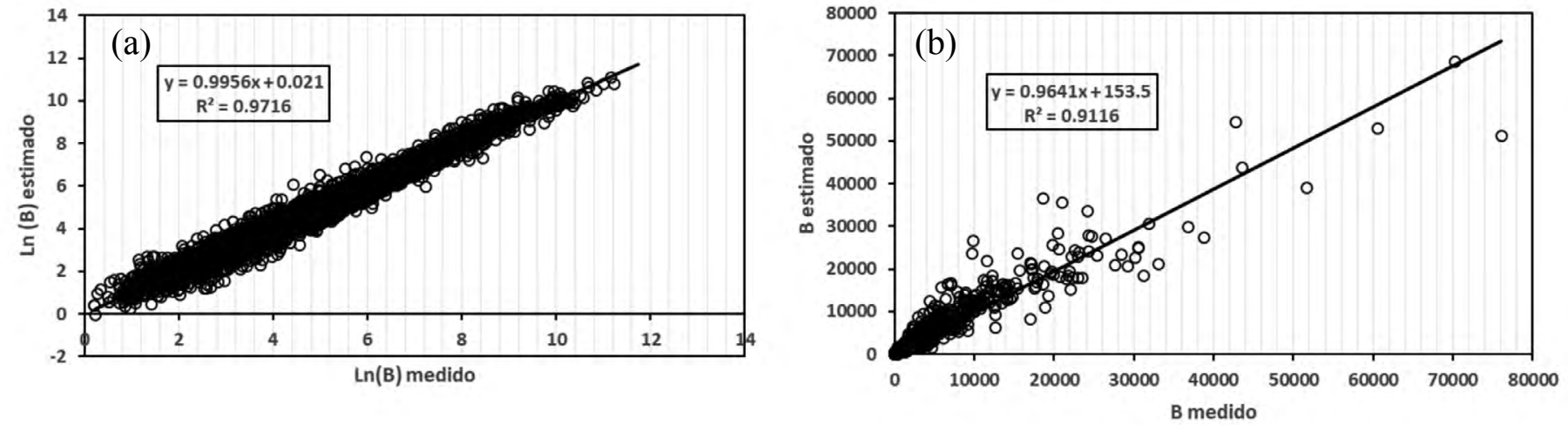

Figura 4. Resultados del ajuste del modelo M3b, función de $\rho$ a nivel de sitio para: (a) espacio logarítmico y (b) espacio aritmético. 
TABla 4. Parámetros y estadísticos de los ajustes en el espacio logarítmico de modelos locales.

\begin{tabular}{llllllll}
\hline Modelo & Parámetros & $q$ & $r$ & $R^{2}$ & ERM & EAM & RECM \\
\hline M1 & Locales & 0.1250 & 0.9738 & 0.9738 & -1.9888 & 8.2688 & 0.3435 \\
M3a & Locales & 0.0306 & 0.9936 & 0.9708 & -1.3119 & 8.5545 & 0.3659 \\
M3b & Ln $\left(a_{\mathrm{vo}}\right)=\operatorname{Ln}(0.0524 p), b_{\mathrm{v}}=1$ & 0.0210 & 0.9956 & 0.9716 & -0.7326 & 7.9350 & 0.3612 \\
M2a & Locales & 0.0905 & 0.9810 & 0.9810 & -1.0681 & 6.5724 & 0.2923 \\
M2b & Locales & -0.0718 & 2.0136 & 0.9570 & 0.6735 & 9.8212 & 0.4564 \\
\hline
\end{tabular}

Tabla 5. Parámetros y estadísticos de los ajustes en el espacio aritmético de los modelos locales.

\begin{tabular}{lllllllll}
\hline Modelo & Parámetros & $F C_{E S}$ & $s$ & $t$ & $R^{2}$ & ERM & EAM & RECM \\
\hline M1 & Locales & Locales & 176.77 & 0.8431 & 0.8501 & -13.8864 & 30.5121 & 1516.9626 \\
M3a & Locales & Locales & 159.03 & 0.9303 & 0.8317 & -16.1245 & 33.4404 & 1663.8007 \\
M3b & $a_{\text {vo }}=0.0524 \rho, b_{\mathrm{v}}=1$ & Locales & 165.04 & 0.9434 & 0.9062 & -14.144 & 31.5702 & 1213.4614 \\
M2a & Locales & Locales & 86.191 & 0.9200 & 0.9371 & -9.2284 & 24.2851 & 984.8277 \\
M2b & Locales & & 48.444 & 0.9593 & 0.9595 & -10.5418 & 35.7043 & 788.0718 \\
\hline
\end{tabular}
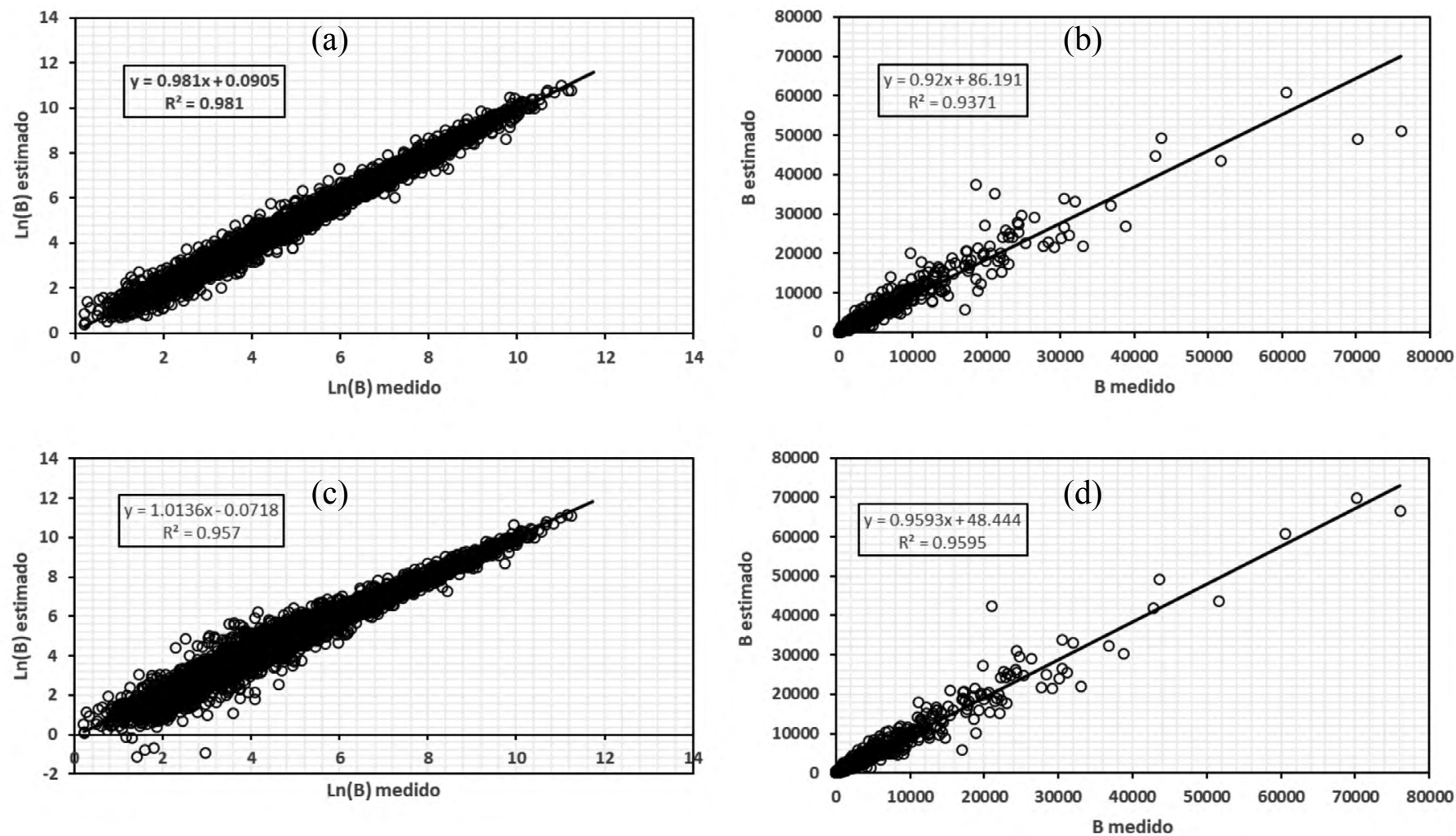

Figura 5. Resultados del proceso de estimación de modelos locales. (a) Modelo M2a en espacio logarítmico, (b) Modelo M2a en espacio aritmético, (c) Modelo M2b en espacio logarítmico y, (d) Modelo M2b en espacio aritmético. 

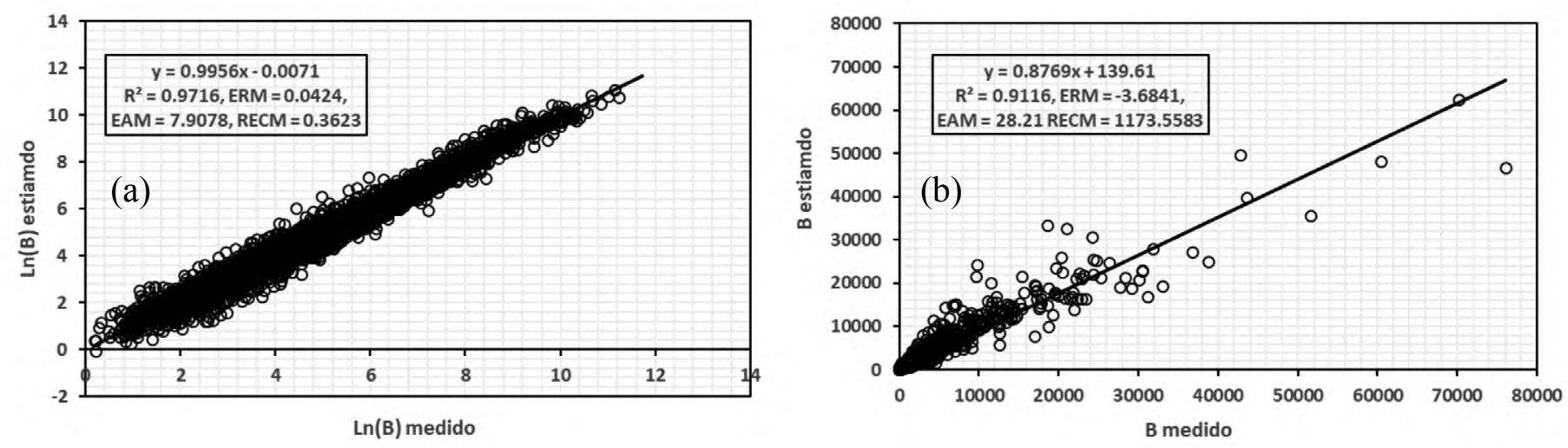

FigURA 6. Resultados del ajuste del modelo M3b usando un proceso de minimización del error absoluto para: (a) espacio logarítmico y (b) espacio aritmético.

\section{Uso del factor de corrección $\mathrm{FC}_{\mathrm{ER}}$}

El uso del factor de corrección $\mathrm{FC}_{\mathrm{ER}}$, relación (11), fue analizado para la conversión de los modelos locales ajustados en el espacio logarítmico al espacio aritmético, particularmente para el caso del modelo M3b que es de solo un parámetro e incorpora la densidad de la madera en forma explícita. La figura 7 muestra los resultados de la aplicación de $\mathrm{FC}_{\mathrm{ER}}$ al modelo $\mathrm{M} 3 \mathrm{~b}$, donde se observa una RECM menor al caso del resto de los modelos, con excepción del M2b de regresión lineal; aunque los valores del error de estimación (RECM) no están alejados del mínimo observado y las métricas ERM y EAM son mejores que el modelo de regresión no lineal.

\section{Modelo empírico para parametrizar $\mathrm{FC}_{\mathrm{ER}}$}

$\mathrm{El}$ factor de corrección $\mathrm{FC}_{\mathrm{ER}}$ requiere del promedio de la biomasa medida, por lo que es necesario estimarla. Aunque la relación entre Best y Bmed está bien caracterizada (Fig. 8), los errores de estimación producen una relación inestable para la estimación de $\mathrm{FC}_{\mathrm{ER}}$. El promedio de la biomasa estimada se refiere a las estimaciones del modelo ajustado en el espacio logarítmico, modelo M3b, convertidas al espacio aritmético usando $\mathrm{FC}=1$ : Best $=\operatorname{Exp}\left[\operatorname{Ln}(0.0595 \rho)+\operatorname{Ln}\left(D^{2} H\right)\right]$.

Una alternativa realizada, como prueba de concepto, fue ajustar un modelo multivariado lineal de regresión estadística para estimar $\mathrm{FC}_{\mathrm{ER}}$, usando para esto datos medidos en campo en los inventarios forestales ( $\mathrm{D}, \mathrm{H}$ y $\rho$ ). El modelo multivariado lineal ajustado a los datos experimentales fue:

$$
\begin{aligned}
& F C_{E R}=1.99-0.963\left\{\frac{\operatorname{Ln}\left[P\left(D^{2} H\right)\right]}{P(\text { Best })}\right\}-0.894\left\{\frac{C V\left(D^{2} H\right)}{C V(\text { Best })}\right\}+2.39619 \mathrm{E}-05\left\{D E\left(D^{2}\right)\right\}- \\
& \text { 1.52984E-05\{DE(Best })\}+0.928\left\{\operatorname{Ln}\left[D E\left(D^{2} H\right)\right]\right\}-0.925\{\operatorname{Ln}[D E(B e s t)]\}+ \\
& 0.448\left\{\frac{D E(D)}{D E(B e s t)}\right\}
\end{aligned}
$$


donde se usaron los operadores $\mathrm{P}$ (promedio), DE (desviación estándar) y CV (coeficiente de variación). La relación (17) es aplicable a nivel de sitio (local).

Los estadísticos del ajuste estadístico multivariado de la relación (17) son: $\mathrm{R}^{2}=0.987, \mathrm{R}^{2}$ ajustada $=0.985, \mathrm{R}^{2}$ predicción $=0.978$, error estándar $=0.024, \mathrm{~d}$ de DurbinWatson $=2.648$, autocorrelación de primer orden $=$
-0.356 , colinealidad $=0.000$, coeficiente de variación $=$ 2.353 .

La figura 9 muestra los resultados del modelo desarrollado usando la relación (17) para estimar $\mathrm{FC}_{\mathrm{ER}}$ a nivel de sitio para el modelo $3 \mathrm{~b}$. Los resultados obtenidos son comparables al uso de $\mathrm{FC}_{\mathrm{ER}}$ medido (Fig. 7).

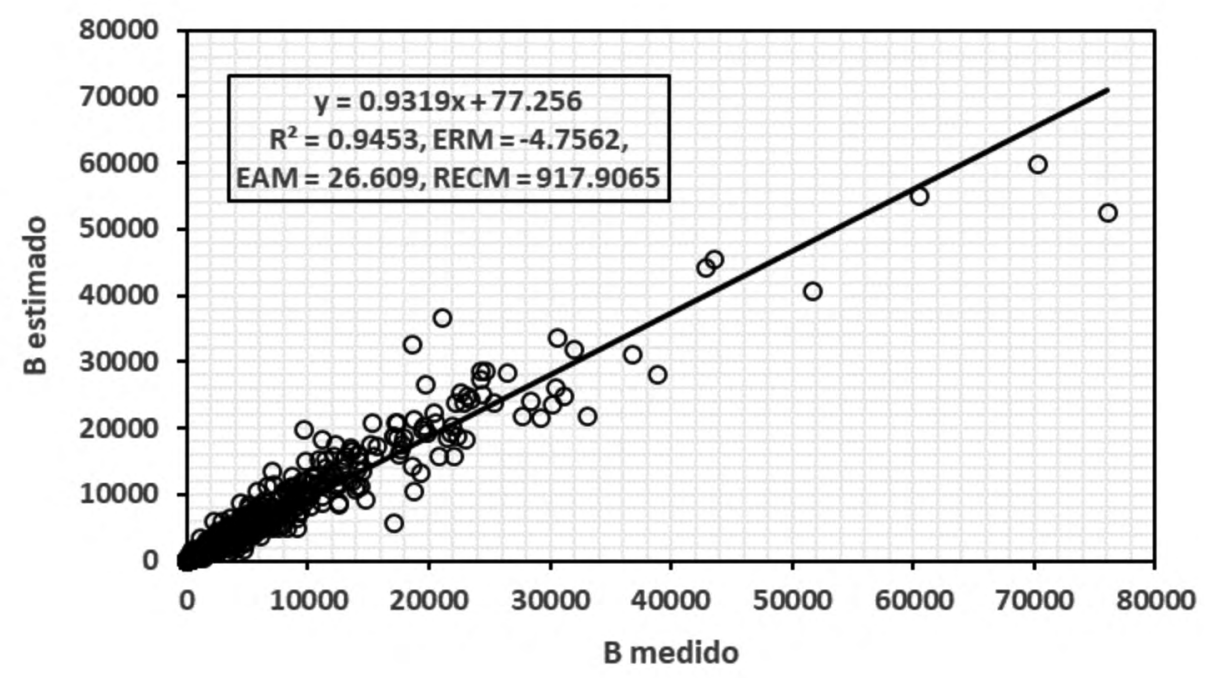

FigURA 7. Resultados del ajuste del modelo $\mathrm{M} 3 \mathrm{~b}$ en el espacio aritmético usando $\mathrm{FC}_{\mathrm{ER}}$ a nivel local.

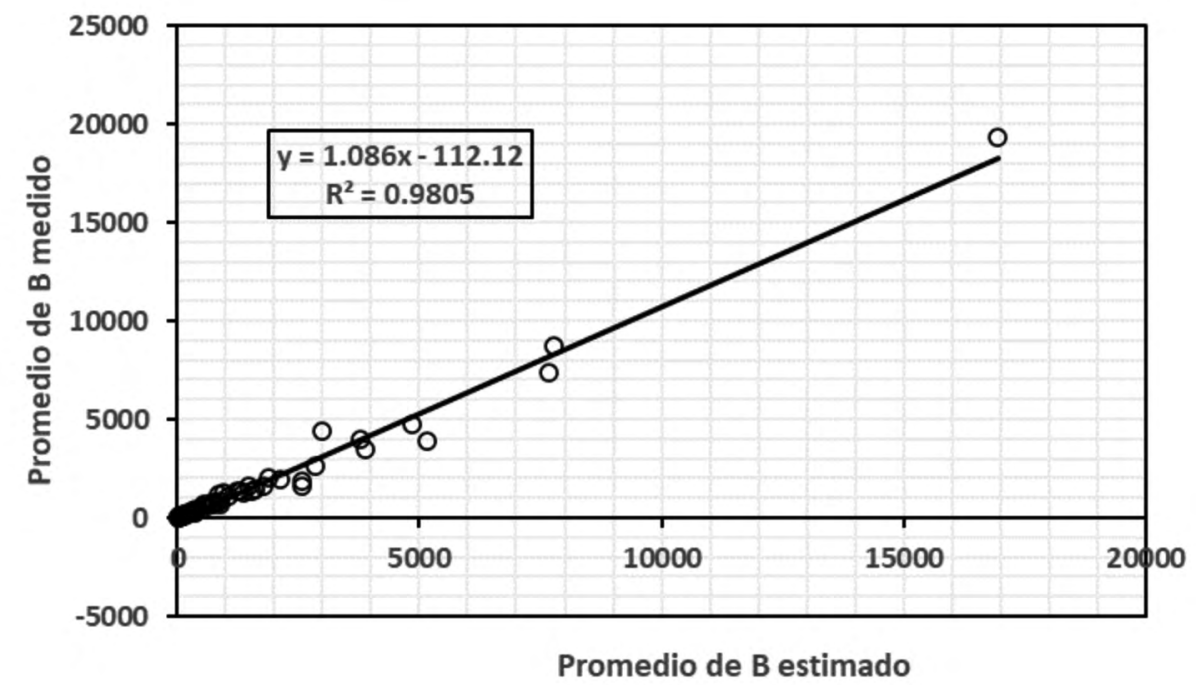

FIGURA 8. Relación entre los promedios de B estimada y B medida de los sitios de la base de datos. 


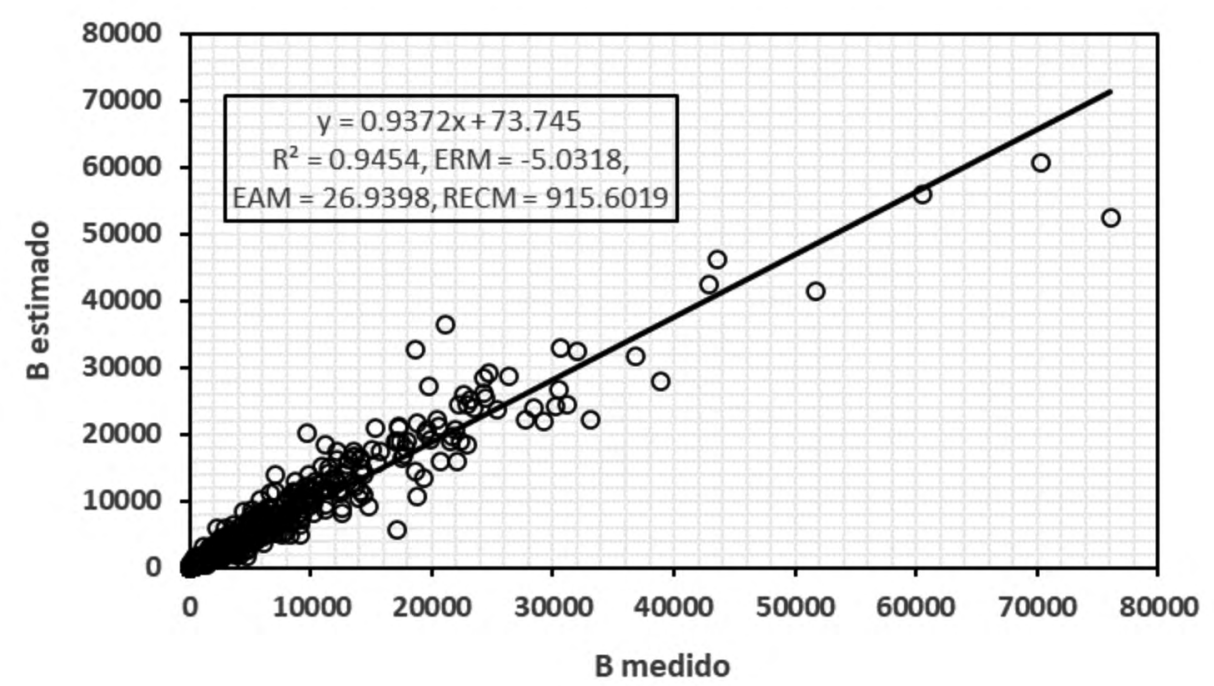

FIGURA 9. Resultados del ajuste del modelo $\mathrm{M} 3 \mathrm{~b}$ en el espacio aritmético usando $\mathrm{FC}_{\mathrm{ER}}$ a nivel local estimado de análisis lineal multivariado.

\section{DISCUSIÓN}

Los diferentes análisis realizados para los ajustes de modelos alométricos en el espacio logarítmico muestran que el objetivo de minimización del error de estimación es de doble paso, ya que también se requiere de la estimación de un factor de corrección. Esta situación está ejemplificada por el modelo de regresión no lineal, donde los resultados del proceso de estimación en el espacio logarítmico son los peores de todos los modelos analizados.

La aproximación clásica de regresión lineal, espacio logarítmico, que minimiza el error cuadrático de estimación (estimación del promedio) fue revisada para considerar otros objetivos, particularmente los sesgos de las estimaciones (error relativo medio y error absoluto medio) por un proceso de minimización del error absoluto. Los resultados mostraron una reducción de los sesgos de estimación con errores de estimación (RECM) comparable al proceso de mínimos cuadrados.

Intentos previos de reducir la dimensionalidad del problema de estimación usando modelo alométricos (Zianis y Mencuccini, 2004; Zianis, 2008; Zhang et al., 2016) han generado resultados mixtos y requerimientos de contar con información de campo normalmente no disponible en los inventarios forestales.
El cambio de factor de corrección simple (Baskerville, 1972) al factor de corrección de estimador de razones de Snowdon (1991), para el caso de estimaciones locales o a nivel de sitio usando el modelo $\mathrm{B}=0.0595 \rho\left(\mathrm{D}^{2} \mathrm{H}\right)$, mejora sustancialmente las estimaciones, aproximándolas a las del modelo de regresión no lineal aplicado a nivel de sitio y con errores de estimación menores al caso de aplicar modelos tipo el usado por Chave et al. (2015) a nivel local, con el uso de factores de conversión clásicos (Baskerville, 1972).

Si se considera que el factor de corrección de Snowdon (1991) requiere de la biomasa medida a nivel de sitio, el desarrollo de un modelo estadístico lineal multivariado usando información disponible en campo, permitió hacer estimaciones comparables al caso de conocer la biomasa medida y con errores de estimación cercanos al modelo de regresión no lineal local que resultó en el mejor modelo.

\section{CONCLUSIONES}

El ideal de desarrollar un modelo alométrico general que permita hacer estimaciones a nivel local, considerando los factores específicos de cada sitio, es uno de los grandes retos en el proceso de estimación de la biomasa aérea, evitando así discusiones sesgadas relacionadas sobre si un 
modelo global es adecuado para las escalas locales, respuesta que es negativa en la gran mayoría de los casos; exceptuando donde la alometría local es similar a la global en todos los casos con el uso de técnicas de regresión estadísticas iguales.

En este trabajo se desarrolló un modelo alométrico generalizado que reduce la dimensionalidad del problema de estimación a un solo parámetro en el espacio logarítmico, pero que requiere un parámetro adicional (factor de corrección) para convertirlo al espacio aritmético usado en las estimaciones de la biomasa aérea. Como prueba de concepto, se desarrolló un modelo estadístico lineal multivariado para estimar el factor de corrección con resultados comparables al caso de conocer la biomasa aérea en cada sitio, requisito para calcular el factor de corrección.

Los resultados obtenidos en este trabajo son altamente promisorios y requieren un análisis de estabilidad de resultados al variar (simulación Monte Carlo) la estructura de las bases de datos de cada sitio (diferentes combinaciones de número de datos y su selección aleatoria); aunque dada la variabilidad de estas estructuras de datos en los sitios de la base de datos pantropical usada permite inferir que la estabilidad es buena.

\section{REFERENCIAS}

Álvarez, E., Duque, Saldarriaga, J., Cabrera, K., De las Salas, G., Del Valle, I., Lema, A., Moreno, F., Orrego, S., \& Rodríguez, L. (2012). Tree above-ground biomass allometries for carbon stocks estimation in the natural forests of Colombia. Forest Ecology and Management, 267(1), 297-308. doi: 10.1016/j.foreco.2011.12.013

Baskerville, G. L. (1972). Use of logarithmic regression in the estimation of plant biomass. Canadian Journal of Forestry, 2(1), 49-53. doi: 10.1139/x72-009

Basuki, T. M., Van Laake, P. E., Skidmore, A. K., \& Hussin, Y. A. (2009). Allometric equations for estimating the aboveground biomass in tropical lowland Dipterocarp forests. Forest Ecology and Management, 257(8), 1684-1694. doi: 10.1016/j.foreco.2009.01.027
Beauchamp, J., \& Olson, J. (1973). Corrections for bias in regression estimates after logarithmic transformation. Ecology, 54(1), 1403-1407. doi: 10.2307/1934208

Bradu, D. \& Mundlak, Y. (1970). Estimation in lognormal linear models. Journal of the American Statistical Association, 65(329), 198-211.doi: 10.2307/2283587

Brown, S. (1997). Estimating biomass change of tropical forests, a primer. Forest Resources Assessment Publication. Forestry Papers 134. Rome: FAO. Recuperado de https://www. fao.org/3/w4095e/w4095e00.htm

Búrquez, A., \& Martínez-Yrízar, A. (2011). Accuracy and bias on the estimation of aboveground biomass in the woody vegetation of the Sonoran Desert. Botany, 89(9), 625633. doi: 10.1139/b11-050

Burt, A., Calders, K., Cuni-Sanchez, A., Gómez-Dans, J., Lewis, P., Lewis, S. L., Mali Y., Phillips, O. \& Disney, M. (2020). Assessment of Bias in Pan-Tropical Biomass Predictions. Frontiers in Forests and Global Change, 3(1), 1-20. doi: 10.3389/ffgc.2020.00012

Cannell, M. G. R. (1984). Woody biomass of forest stands. Forest Ecology and Management, 8(3-4), 299-312. doi:10.1016/0378-1127(84)90062-8

Chave, J., Andalo, C., Brown, S., Cairns, M. A., Chambers, J. Q., Eamus, D., Fölster, H., Fromard, F., Higuchi, N., Kira, T., Lescure, J. P., Nelson, B. W., Ogawa, H., Puig, H., Riéra, B., \& Yamakura, T. (2005). Tree allometry and improved estimation of carbon stocks and balance in tropical forests. Oecologia, 145(1), 87-99. doi: 10.1007/ s00442-005-0100-x

Chave, J., Coomes, D., Jansen, S., Lewis, S. L., Swenson, N. G., \& Zanne, A. E. (2009). Towards a worldwide wood economics spectrum. Ecology Letters, 12(4), 351-366. doi: 10.1111/j.1461-0248.2009.01285.x

Chave, J., Réjou-Méchain, M., Búrquez, A., Chidumayo, E., Colgan, M. S., Delitti, W. B. C., Duque, A., Eid, T., Fearnside, P. M., Goodman, R. S., Henry ,M., MartínezYrízar, A., Mugasha, W. A., Muller-Landau, H. C., Men- 
cuccini, M., Nelson, B. W., Ngomanda, A., Nogueira, E. M., Ortiz-Malavassi, E., Pélissier, R., Ploton, P., Ryan, C. M., Saldarriaga, J. G., \& Vieilledent, G. (2014). Improved allometric models to estimate the aboveground biomass of tropical trees. Global Change Biology, 20(10), 31773190. doi:10.1111/gcb.12629

Clifford, D., Cressie, N., England, J. R., Roxburgh, S. H., \& Paul, K. I. (2013). Correction factors for unbiased, efficient estimation and prediction of biomass from log-log allometric models. Forest Ecology and Management, 310, 375-381. doi: 10.1016/j.foreco.2013.08.041

Duan, N. (1983). Smearing Estimate: A Nonparametric Retransformation Method. Journal of the American Statistical Association, 78(383), 605. doi:10.2307/2288126

El-Shaarawi, A. H., \& Viveros, R. (1997). Inference About the Mean in Log-Regression with Environmental Applications. Environmetrics, 8(5), 569-582. doi:10.1002/ (S IC I ) 1099 - 095 X(199709/10)8:5<569:: A ID ENV274>3.0.CO;2-I

Finney, D. J. (1941). On the Distribution of a Variate Whose Logarithm is Normally Distributed. Supplement to the Journal of the Royal Statistical Society, 7(2), 155-161. doi:10.2307/2983663

Forrester, D. I., Tachauer, I. H. H., Annighoefer, P., Barbeito, I., Pretzsch, H., Ruiz-Peinado, R., Stark, H., Vacchiano, G., Zlatanov, T., Chakraborty, T., Saha, S., \& Sileshi, G. W. (2017). Generalized biomass and leaf area allometric equations for European tree species incorporating stand structure, tree age and climate. Forest Ecology and Management, 396, 160-175. doi: 10.1016/j. foreco.2017.04.011

Genet, A., Wernsdörfer, H., Jonard, M., Pretzsch, H., Rauch, M., Ponette, Q., Nys, C., Legout, A., Ranger, J., Vallet, L., \& Saint-André, L. (2011). Ontogeny partly explains the apparent heterogeneity of published biomass equations for Fagus sylvatica in central Europe. Forest Ecology and Management, 261(7), 1188-1202. doi: 10.1016/j. foreco.2010.12.034

Henry, M., Picard, N., Trotta, C., Manlay, R. J., Valentini, R., Bernoux, M., \& Saint-André, L. (2011). Estimating tree biomass of Sub-Saharan African forests: a review of avai- lable allometric equations. Silva Fennica, 45(3B), 477569. doi: 10.14214/sf.38

Hui, C., Terblanche, J. S., Chown, S. L., \& McGeoch, M. A. (2010). Parameter landscapes unveil the bias in allometric prediction. Methods in Ecology and Evolution, 1(1), 69-74. doi: 10.1111/j.2041-210X.2009.00005.x

Jenkins, J. C., Chojnacky, D. C.,Heath, L. S., \& Birdsey, R. A. (2003). National-scale biomass estimators for United States tree species. Forest Science, 49(1), 12-35.

Journel, A.G. (1984). mAD and conditional quantile estimators. In: G. Verly, M. David, A.G. Journel and A. Marechal (eds.). Geostatistics for Natural Resources Characterization, (pp. 261-270). Part 1. D. Reidel Publishing Company. Dordrecht. doi: 10.1007/978-94-009-3699-7_16

Lee, C. Y. (1982). Comparison of two correction methods for the bias due to the logarithmic transformation in the estimation of biomass. Canadian Journal of Forest Research, 12(2), 326-331. doi:10.1139/x82-047

Manuri, S., Brack, C., Noor'an, F., Rusolono, T., Anggraini, S. M., Dotzauer, H., \& Kumara, I. (2016). Improved allometric equations for tree aboveground biomass estimation in tropical dipterocarp forests of Kalimantan, Indonesia. Forest Ecosystems, 3(1). doi: 10.1186/s40663016-0087-2

Méndez González J., Turlan Medina, O. A., Ríos Saucedo J. C., \& Nájera Luna J. A. (2012). Ecuaciones alométricas para estimar biomasa aérea de Prosopis laevigata (Humb. and Bonpl. Ex Willd.) m. C. Johnst. Revista Mexicana de Ciencias Forestales, 3(13), 57-72. doi: 10.29298/rmcf. v3i13.489

Ngomanda, A., Obiang, N., Lebamba, J., Mavouroulou, Q., Gomat, H., Mankou, G., Loumeto, J., Iponga, D., Ditsouga, F., Koumba, C. R., Henga-Botsikabobe, K., Okouyi, C., Nyangadouma, R., Lepengue, A., Mbatchi, B. \& Picard, N. (2014). Site-specific versus pantropical allometric equations: Which option to estimate the biomass of a moist central African forest? Forest Ecology and Management, 312(1), 1-9. doi: 10.1016/j. foreco.2013.10.029 
Packard, G. C. (2017). Misconceptions about logarithmic transformation and the traditional allometric method. Zoology, 123(1), 115-120. doi: 10.1016/j.zool.2017.07.005

Paul, K. I., Roxburgh, S.H., Chave, J., England, J. R., Zerihun, A., Specht, A., Lewis, T., Bennet, L. T., Baker, T. G., Adams, M. A., Huxtable, D., Montagu, K. D., Falster, D. S., Feller, M., Sochacki, S., Ritson, P., Bastin, G., Bartle, J., Wildy, D., Hobbs, T., Larmour, J., Waterworth, R., Stewart, H. T. L., Jonson, J., Forrester, D. I., Applegate, G., Mendham, D., Bradford, M., O’Grady, D. A., Green, D., Sudmeyer, R., Rance, S. J., Turner, J., Barton, C., Wenk, E. H., Grove, T., Attiwill, P. M., Pinkard, E., Butler, D., Brooksbank, K., Spencer, B., Snowdon, P., O’Brien, N., Battaglia, M., Cameron, D. M., Hamilton, S., McAuthur, G. \& Sinclair, J. (2016). Testing the generality of above-ground biomass allometry across plant functional types at the continental scale. Global Change Biology, 22(6): 2106-2124. doi: 10.1111/gcb.13201

Pelletier, J., Kirby, K. R., \& Potvin, C. (2012). Significance of carbon stock uncertainties on emission reductions from deforestation and forest degradation in developing countries. Forest Policy and Economics, 24(1), 3-11. doi: 10.1016/j.forpol.2010.05.005

Picard, N., F. Boyemba B., \& Rossi, V. (2015). Reducing the error in biomass estimates strongly depends on model selection. Annals of Forest Science, 72(1), 811-823. doi: 10.1007/s13595-014-0434-9

Ploton, P., Barbier, N., Takoudjou Momo, S., Réjou-Méchain, M., Boyemba Bosela, F., Chuyong, G., Dauby, G., Droissart, V., Fayolle, A., Goodman, R. C., Henry, M., Kamdem, N. G., Mukirania, J. K., Kenfack, D., Libalah, M., Ngomanda, A., Rossi, V., Sonké, B., Texier, N., Thomas, D., Zebaze, D., Couteron, P., Berger, U., and Pélissier, R. (2016). Closing a gap in tropical forest biomass estimation: taking crown mass variation into account in pantropical allometries. Biogeosciences 13(1), 1571-1585. doi: 10.5194/bg-13-1571-2016

Rojas-García, F., De Jong, B. H. J., Martínez-Zurimendí, P., \& Paz-Pellat, F. (2015). Database of 478 allometric equations to estimate biomass for Mexican trees and forests.
Annals of Forest Science, 72(6), 835-864. doi:10.1007/ s13595-015-0456-y

Sato, T., Saito, M., Ramírez, D., Pérez De Molas, L. F., Toriyama, J., Monda, Y., Kiyono, Y., Herebia, E., Dubie, N., Duré Vera, E., Ramírez Ortega, J. D., \& Vera De Ortiz, M. (2015). Development of Allometric Equations for Tree Biomass in Forest Ecosystems in Paraguay. Japan Agricultural Research Quarterly, 49(3), 281-291. doi: 10.6090/ jarq.49.281

Shen, H., \& Zhu, Z. (2008). Efficient mean estimation in lognormal linear models. Journal of Statistical Planning and Inference, 138(3), 552-567. doi: 10.1016/j. jspi.2006.10.016

Smith, R. J. (1993). Logarithmic transformation bias in allometry. American Journal of Physical Anthropology, 90(2), 215-228. doi: 10.1002/ajpa.1330900208

Snowdon, P. (1991). A ratio estimator for bias correction in logarithmic regressions. Canadian Journal of Forest Research, 21(5), 720-724. doi: 10.1139/x91-101

Temesgen, H., Affleck, D., Poudel, K., Gray, A., \& Sessions, J. (2015). A review of the challenges and opportunities in estimating above ground forest biomass using tree-level models. Scandinavian Journal of Forest Research, 30(4),1-10. doi: 10.1080/02827581.2015.1012114

Van Breugel, M., Ransijn, J., Craven, D., Bongers, F., \& Hall, J. S. (2011). Estimating carbon stock in secondary forests: Decisions and uncertainties associated with allometric biomass models. Forest Ecology and Management, 262(8), 1648-1657. doi: 10.1016/j.foreco.2011.07.018

Vargas-Larreta, B., López-Sánchez, C. A., Corral-Rivas, J. J., López-Martínez, J. O., Aguirre-Calderón, C. G., \& Álvarez-González, J. G. (2017). Allometric Equations for Estimating Biomass and Carbon Stocks in the Temperate Forests of North-Western Mexico. Forests, 8(8), 269. doi: 10.3390/f8080269

Wutzler, T., Wirth, C., \& Schumacher, J. (2008). Generic biomass functions for Common beech (Fagus sylvatica) in Central Europe: predictions and components of uncertainty. Canadian Journal of Forest Research, 38(6), 1661-1675. doi: 10.1139/x07-194 
Zanne, A.E., Lopez-Gonzalez, G., Coomes, D.A., Jansen, S., Lewis, L.S., Miller, R.B., Swenson, N.G., Wiemann, M.C., \& Chave, J. Data from: Towards a wordwide Wood economics spectrum, V5, Dryad, Dataset. doi: 10.506/ dryad. 234

Zar, J. H. (1968). Calculation and Miscalculation of the Allometric Equation as a Model in Biological Data. BioScience, 18(12), 1118-1120. doi: 10.2307/1294589

Zeng, W. S., \& Tang, S. Z. (2011). Bias Correction in Logarithmic Regression and Comparison with Weighted Regression for Nonlinear Models. Nature Precedings. doi:10.1038/npre.2011.6708.1

Zhang, Z., Zhong, Q., Niklas, K. J., Cai, L., Yang, Y., \& Cheng, D. (2016). A predictive nondestructive model for the covariation of tree height, diameter and stem volume scaling relationships. Scientific Reports, 6(1), 1-9. doi: 10.1038/ srep 31008
Zianis, D. (2008). Predicting mean aboveground forest biomass and its associated variance. Forest Ecology and Management, 256(6), 1400-1407. doi: 10.1016/j. foreco.2008.07.002

Zianis, D., \& Mencuccini, M. (2004). On simplifying allometric analyses of forest biomass. Forest Ecology and Management, 187(2-3), 311-332. doi: 10.1016/j. foreco.2003.07.007

Manuscrito recibido el 19 de mayo de 2021

Aceptado el 12 de octubre de 2021

Publicado el 02 de diciembre de 2021

Este documento se debe citar como:

Paz-Pellat, F. (2021). Un modelo alométrico pantropical global y local. Madera y Bosques, 27(4), e2742446. doi: 10.21829/myb.2021.2742446

Madera y Bosques, por Instituto de Ecología, A.C. se distribuye bajo una Licencia Creative Commons Atribución-No Comercial-Compartir Igual 4.0 Internacional. 